\title{
PREVALENCIA Y NIVELES DE EXPOSICIÓN A FACTORES Y RIESGOS PSICOSOCIALES A TRAVÉS DEL ISTAS-21
}

\section{Prevalence and exposure levels to psychosocial factors and risks through ISTAS-21}

\author{
Esmeralda Montalvo SÁncheZ \\ Universidad de Extremadura \\ Eloísa Guerrero Barona \\ Universidad de Extremadura \\ eloisa@unex.es \\ Marta RodRígueZ JimÉNEZ \\ Universidad de Extremadura \\ Jonás Agudo Osuna \\ Servicio Mancomunado de Prevención de Riesgos Laborales de FEAPS ${ }^{1}$ Extremadura (SEMFE) \\ Juan Manuel Moreno Manso \\ Universidad de Extremadura \\ Daniel PARedes Gómez \\ Centro Ocupacional Aprosuba-4 (Extremadura)
}

Recepción: 6 de marzo de 2019

Aceptación definitiva: 25 de noviembre de 2019

Resumen: El presente trabajo tiene como finalidad la evaluación y descripción de los riesgos psicosociales en una muestra de profesionales de Extremadura que prestan servicios de atención a personas con discapacidad intelectual o del desarrollo. Para ello, se ha utilizado el cuestionario Istas-21 (ISTAS, 2010). La muestra está formada por 518 trabajadores y trabajadoras, 169 son hombres $(30,11 \%)$ y 349 son mujeres $(67,37 \%)$

1 Federación de Asociaciones en favor de las Personas con Discapacidad Intelectual de Extremadura. Es una organización no gubernamental y sin ánimo de lucro que presta servicios sociales especializados a las personas con discapacidad y a sus familias. 
PREVALENCIA Y NIVELES DE EXPOSICIÓN A FACTORES Y RIESGOS PSICOSOCIALES A TRAVÉS DEL ISTAS-21 ESMERALDA MONTALVO SÁNCHEZ, ELOÍSA GUERRERO BARONA, MARTA RODRÍGUEZ JIMÉNEZ, JONÁS AGUDO OSUNA, JUAN MANUEL MORENO MANSO Y DANIEL PAREDES GÓMEZ

de diferentes entidades. Se han analizado tres constructos psicosociales: exigencias psicológicas, doble presencia y control del trabajo, en función de la edad, sexo, departamento, antigüedad, puesto de trabajo y tipo de contrato. Estos constructos engloban 10 dimensiones del cuestionario que se han analizado pormenorizadamente. Los resultados indican que los participantes están más afectados por las dimensiones de las exigencias de esconder emociones, doble presencia y control de los tiempos en el trabajo.

Palabras clave: factores psicosociales; riesgos psicosociales; discapacidad intelectual e ISTAS-21.

Aвstract: The main purpose of the present study is to evaluate and to explain the presence of psychosocial risks in a cross-section of staff working with people with intellectual disabilities from Extremadura (Spain). With this aim, Istas-21 instrument has been used. The cross-section was composed of 518 workers of different entities, 169 are men $(30,11 \%)$ and 349 are women $(67,37 \%)$. Three psychosocial constructs of Istas-21 have been analyzed: psychosocial demands, double presence and time management, considering different variables (age, sex, department, numbers of years spent on the work, job position and type of contract). These constructs include ten dimensions of the questionnaire which have been deeply evaluated. The results indicate that participants are more affected by the dimensions of the demands of hiding emotions, double presence and time management at work.

KEY WORDS: psychosocial factors; psychosocial risks; intellectual disability and ISTAS-21.

\section{Introducción}

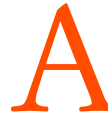

CTUALMENTE ESTAMOS VIVIENDO una situación económica muy desfavorable en nuestro país, lo cual está afectando de forma directa al mercado laboral (Ramos y Peiró, 2014). Una de las principales pérdidas son los derechos de los empleados/as en sus puestos de trabajo y ello ha generado numerosos estudios sobre los factores y riesgos psicosociales que afectan al ámbito laboral. Estos cambios conllevan nuevas organizaciones laborales, que exigen a los profesionales y a las profesionales una gran capacidad de adaptación y de afrontamiento a las situaciones laborales (Guerrero, Gómez, Moreno, García-Baamonde y Blázquez, 2011).

El presente trabajo se contextualiza en la Psicología de la Salud Ocupacional, disciplina que aborda el estudio de los riesgos psicosociales en el trabajo, el estrés laboral y la calidad de vida en el trabajo. No se pueden definir con rigor los riesgos psicosociales si no clarificamos qué son las condiciones laborales y/o los factores psicosociales. Hay confusión en estos tres términos, y por esta razón la primera parte de esta sección se dedicará a diferenciarlos y posteriormente los vincularemos a sus orígenes, esto es, a la Psicosociología. El término psicosocial hace referencia a la conducta humana y a su integración en la sociedad analizada desde los aspectos sociales. La Psicosociología o Psicología social aplicada estudia aspectos de la Psicología social

Ediciones Universidad de Salamanca / CC BY-NC-ND

Siglo Cero, vol. 51 (1), 2020, enero-marzo, pp. 53-72 
PREVALENCIA Y NIVELES DE EXPOSICIÓN A FACTORES Y RIESGOS PSICOSOCIALES A TRAVÉS DEL ISTAS-21

ESMERALDA MONTALVO SÁNCHEZ, ELOÍSA GUERRERO BARONA, MARTA RODRÍGUEZ JIMÉNEZ, JONÁS AGUDO OSUNA, JUAN MANUEL MORENO MANSO Y DANIEL PAREDES GÓMEZ

y los aplica para fines sociales, mientras que la Psicosociología aplicada en materia de prevención de riesgos laborales es una materia académica que aplica los conocimientos de la Salud Ocupacional para la evaluación, prevención y tratamiento de los riesgos psicosociales en el trabajo (Gil-Monte, 2014).

Desde la Ley de Prevención de Riesgos Laborales se definen las condiciones de trabajo como "cualquier característica del trabajo que puede tener una influencia significativa en la generación de riesgos para la seguridad y la salud del trabajador" (LPRL, 31/1195, art. 4.7, p. 2). A partir de ella, para algunos autores, condiciones de trabajo, condiciones organizacionales o factores psicosociales son términos intercambiables (Gil-Monte, 2012; Moreno y Garragosa, 2013).

En línea con lo anterior, otros autores han afirmado que condiciones de trabajo son todos aquellos factores laborales que pueden afectar a la salud del trabajador (Jodar y Benaviders, 2006) y en esta consideración existe bastante unanimidad. En este sentido, Gil-Monte (2012) afirma que cuando las condiciones de trabajo o los factores psicosociales perjudican y afectan a la actividad y calidad de vida laboral se habla de riesgos psicosociales. Sin embargo, para este mismo autor, también los factores psicosociales pueden favorecer la actividad laboral y ser fuente de satisfacción y desarrollo personal.

Posteriormente, Gil-Monte (2014) definió con más precisión los riesgos psicosociales como "aquellos aspectos del diseño del trabajo, la organización y sus contextos sociales que tienen el potencial de causar daño a la persona, ocasionado por un deterioro o disfunción en la tarea, la organización, las características del empleo o la organización del tiempo de trabajo” (p. 30). Por tanto, los riesgos psicosociales tienen el potencial de causar daño psicológico, físico o social a los individuos enfatizando que su origen está en el entorno y no en el individuo, aunque evidentemente habrá individuos que en las mismas condiciones laborales respondan de distinto modo.

A lo largo de este trabajo entenderemos que los factores psicosociales son las condiciones de trabajo que, una vez evaluadas por la persona, pueden influir positiva o negativamente sobre su salud, dependiendo de la valoración y el significado que la persona concede a esa situación, percibiéndola como una situación inocua o no amenazante o, por el contrario, como amenazante o como situación de riesgo físico, psicológico y/o social. Sin embargo, los riesgos psicosociales siempre se relacionarán con evaluaciones amenazantes o peligrosas y con condiciones de trabajo difíciles de tolerar para la mayoría de los individuos.

Los riesgos psicosociales tienen características propias, como la existencia de cobertura legal, en caso del síndrome de burnout o del acoso laboral, debido a que son infringidos derechos fundamentales como intimidad, igualdad, dignidad o libertad (Moreno y Báez, 2010). También a largo plazo pueden causar enfermedades psicofisiológicas (cardiovasculares, respiratorias, inmunitarias, gastrointestinales, dermatológicas, endocrinológicas, musculoesqueléticas) y mentales. Una característica de los riesgos psicosociales es su frecuente influencia sobre la salud mental. El estrés agudo, la depresión y los trastornos de ansiedad suelen ser consecuencias de los riesgos psicosociales laborales (Moreno-Jiménez y Garragosa, 2013).

Desde otros planteamientos, los riesgos psicosociales se pueden clasificar, basándose en el proceso de estrés, en dos grandes bloques: como demandas laborales 
PREVALENCIA Y NIVELES DE EXPOSICIÓN A FACTORES Y RIESGOS PSICOSOCIALES A TRAVÉS DEL ISTAS-21

ESMERALDA MONTALVO SÁNCHEZ, ELOÍSA GUERRERO BARONA, MARTA RODRÍGUEZ JIMÉNEZ, JONÁS AGUDO OSUNA, JUAN MANUEL MORENO MANSO Y DANIEL PAREDES GÓMEZ

o estresores o como falta de recursos personales y laborales (Schaufeli y Salanova, 2002). Desde esta perspectiva, los estresores o demandas laborales se entienden como aspectos físicos, sociales y organizacionales que requieren esfuerzo sostenido y se encuentran asociados a costes fisiológicos y psicológicos. Los recursos personales y laborales, en cambio, se asocian a aspectos físicos, psicológicos y sociales y organizacionales que son funcionales para la consecución de objetivos, reducen las exigencias laborales y potencian el desarrollo personal.

Desde la Psicosociología, el estrés se concibe como un desequilibrio percibido entre las demandas del medio y la falta de recursos de la persona para afrontarlas. La sensación de estrés la estimulan los desencadenantes o estresores. En el contexto laboral, los estresores también pueden denominarse riesgos psicosociales (Gil-Monte, 2014). Para que los riesgos psicosociales o estresores generen una respuesta de estrés deben ser percibidos por el individuo como amenazantes o como estímulos peligrosos. Siguiendo a este mismo autor, el estrés se puede definir como una transacción entre la persona y el ambiente resultante de la valoración que la persona hace de dicha situación y es por ello importante considerar la mediación de variables personales.

Del mismo modo, los estresores pueden tener como efectos consecuencias negativas para el individuo. Entre las consecuencias, cabe destacar el síndrome de burnout o síndrome de quemarse por el trabajo (Gil-Monte, 1999; Salanova, Schaufeli, Llorens, Grau y Peiró, 2000). El síndrome de burnout es concebido como una respuesta a fuentes de estrés laboral crónico vinculadas a relaciones sociales entre los profesionales de servicios y a los receptores de los mismos, que aparece cuando fallan las estrategias de afrontamiento al estrés. En este caso, el burnout funciona como una variable mediadora entre la percepción de fuentes de estrés crónico y sus consecuencias (Schaufeli y Salanova, 2002).

La Organización Internacional de Trabajo propuso en 1986 un primer listado de los factores psicosociales, que incluía una baja utilización de las habilidades, la desigualdad del salario, la falta de seguridad en el trabajo, los problemas en las relaciones laborales, el trabajo por turnos, el peligro físico, la sobrecarga de trabajo, el conflicto de autoridad y la falta de control sobre el trabajo.

Igualmente, Moreno-Jiménez y Báez (2010) analizaron los factores propuestos por Roozeboom, Houtman y Van de Bossce, y los clasificaron en función de dos indicadores: organizacionales y laborales. En los factores organizacionales se incluyen la política y filosofía de la organización, la cultura de la misma y las relaciones industriales, mientras que en los factores laborales se encuentran las condiciones del empleo, el diseño del puesto y la calidad en el trabajo.

Las características de los factores psicosociales de riesgo son muy relevantes para comprender el origen de los mismos, son variadas y dependen de los puestos de trabajo (Moreno-Jiménez y Báez, 2010). Algunas de sus principales características se refieren a que estos factores se extienden en el espacio y en el tiempo, diferenciándose por la dificultad de objetivación por parte de los empleados/as y por la cobertura legal que se les ofrece, motivos por los cuales su intervención es compleja.

Aunque muchos autores utilizan indistintamente los factores y riesgos psicosociales, Gil-Monte (2012) puntualiza que los riesgos psicosociales son un deterioro de 
PREVALENCIA Y NIVELES DE EXPOSICIÓN A FACTORES Y RIESGOS PSICOSOCIALES A TRAVÉS DEL ISTAS-21

ESMERALDA MONTALVO SÁNCHEZ, ELOÍSA GUERRERO BARONA, MARTA RODRÍGUEZ JIMÉNEZ, JONÁS AGUDO OSUNA, JUAN MANUEL MORENO MANSO Y DANIEL PAREDES GÓMEZ

las características de la tarea, de la organización, del empleo y del tiempo de trabajo. Algunas investigaciones revelan que los principales riesgos psicosociales que sufren los individuos en sus puestos de trabajo son el estrés, burnout, violencia en el trabajo, acoso laboral, acoso sexual, inseguridad laboral, conflicto trabajo-familia y trabajo emocional (Moreno-Jiménez y Báez, 2010 y Mansilla, García, Gamero y Congosto, 2010).

En referencia a las consecuencias de la existencia de estos riesgos psicosociales, numerosos estudios llevados a cabo en los últimos años muestran resultados muy significativos, a través de los cuales podemos ver la situación actual en la que se encuentra el personal en sus puestos de trabajo. Así, la VII Encuesta Nacional de Condiciones del Trabajo (2012) destaca que siete de cada diez trabajadores/as tienen problemas de salud relacionados con sus puestos de trabajo. También la Agencia Europea para la Seguridad y Salud Ocupacional (EU-OSHA, 2009) indica que el $22 \%$ de los trabajadores sufren estrés. Este riesgo representa el $50 \%$ de días perdidos de trabajo en Europa. Igualmente, esta agencia ha realizado una encuesta de opinión paneuropea sobre seguridad y salud en el trabajo en 31 países europeos. En España se llevaron a cabo 501 entrevistas (EU-OSHA, 2013), concluyendo que las causas principales por las cuales los sujetos sufren estrés son la carga de trabajo, la inseguridad laboral y estar expuestos a conductas inaceptables, como el acoso y la intimidación.

Otro aspecto relevante se refiere a la creación de instrumentos válidos y fiables para valorar la presencia de riesgos psicosociales y cuya finalidad es la eliminación o prevención de los mismos en los puestos de trabajo. Dicha prevención ha quedado recogida en nuestro país en la Ley de Prevención de Riesgos Laborales (INSHT, 1995). En España los estudios sobre los riesgos psicosociales se han centrado principalmente en el desarrollo de instrumentos de evaluación y de análisis, en el estudio de diferentes modelos teóricos y en la investigación sobre la eficacia de las medidas de prevención en las empresas (Peiró, Yeves y Lorente, 2013).

Existe un gran listado de instrumentos válidos y fiables para evaluar los riesgos psicosociales. Muchos de ellos han sido elaborados por los institutos dedicados a la salud laboral. Algunos de los más utilizados son F-PSICO, desarrollado por el Instituto Nacional de Salud e Higiene en el trabajo (Martín y Pérez, 1997); el Cuestionario multidimensional DECORE, elaborado por la Universidad Complutense de Madrid (Luceño, Martín, Jaén y Díaz, 2005), y finalmente el Método Istas-21, que es el que se ha utilizado en el presente estudio. Sus características psicométricas se analizarán detalladamente en la sección de Instrumentos. La elección se ha basado en las numerosas ventajas que presenta, ya que es un instrumento de uso público y gratuito, dispone de varias versiones disponibles para las distintas empresas y puede ser aplicado por técnicos en prevención de riesgos psicosociales sin ser expertos en conducta organizacional.

A pesar de las investigaciones desarrolladas y las medidas de prevención en los diferentes puestos laborales, son muchos los grupos de profesionales que están sometidos a los riesgos psicosociales. En este sentido, el personal del sector servicio asistencial es el que presenta un alto riesgo de sufrir las consecuencias del sindrome de burnout (Moriana, Alos, Pino, Herruzo, Ruiz, Alcalá y Corpas, 2006) y está continuamente trabajando en condiciones duras con otras personas (Gil-Monte 
PREVALENCIA Y NIVELES DE EXPOSICIÓN A FACTORES Y RIESGOS PSICOSOCIALES A TRAVÉS DEL ISTAS-21

ESMERALDA MONTALVO SÁNCHEZ, ELOÍSA GUERRERO BARONA, MARTA RODRÍGUEZ JIMÉNEZ, JONÁS AGUDO OSUNA, JUAN MANUEL MORENO MANSO Y DANIEL PAREDES GÓMEZ

y Figueiredo, 2013). Así mismo, este colectivo es considerado de alto riesgo por sus condiciones de trabajo, como por ejemplo falta de apoyo social por parte de los compañeros, un bajo significado de su tarea, ambigüedad y conflicto de rol, falta de autonomía o incluso agresiones de los usuarios a los que atiende (Gil-Monte, Carretero, Roldán y Núñez-Román, 2005). Además, el riesgo de sufrir los efectos de los riesgos psicosociales y burnout puede ser disminuido cuando los trabajadores cuentan con apoyo social, tienen control sobre su trabajo y una percepción positiva de la competencia personal, es decir, las competencias cognitivas, sociales y aptitudinales pueden tener un efecto mediador y ayudar a prevenir los efectos de los factores y riesgos psicosociales (Avargues, Borda y López, 2010).

En referencia a la población específica de trabajadores con personas con discapacidad intelectual, estudios llevados a cabo en esta población han puesto de manifiesto que muchos de estos profesionales se encuentran sometidos a altos niveles de estrés (e. g. Mutkins, Brown y Thorsteinsson, 2011). Entre los riesgos psicosociales identificados y que se asocian a la ocurrencia de burnout se encuentran: ausencia de apoyo organizacional y de apoyo social (Mutkins et al., 2011), sobrecarga en el trabajo, ausencia de participación en la toma de decisiones (Gray-Stanley y Muramatsu, 2011), los conflictos familia-trabajo, trabajo emocional o los conflictos de rol (Kozak, Kersten, Schillmoler y Nienhaus, 2013). El padecimiento de burnout en trabajadores con personas con discapacidad intelectual ha sido asociado con la intención de abandonar el empleo, mientras que niveles disminuidos se relacionan con un incremento en la salud de los trabajadores, con una mayor satisfacción con el trabajo y, además, con una mayor satisfacción con la vida en general (Kozak et al., 2013).

Debido a que, como se observa, la presencia de riesgos psicosociales en el trabajo se asocia con importantes consecuencias, en este trabajo nos proponemos analizar y evaluar los principales factores y riesgos psicosociales en una muestra de trabajadores que atienden a personas con discapacidad intelectual para identificar aquellos factores y riesgos psicosociales que se encuentran presentes y que, potencialmente, pueden afectar a su salud y a su bienestar.

La novedad de este trabajo radica en que por primera vez se aplica la evaluación de factores y riesgos psicosociales a profesionales de servicios, que atienden a personas con discapacidad en la Comunidad Autónoma Extremeña, un colectivo vulnerable a riesgos laborales.

La relevancia de este estudio radica en aportar evidencias científicas que permitan posteriormente diseñar, desarrollar y aplicar medidas y programas de prevención e intervención dirigidos al bienestar y salud personal y organizacional y a la mejora de la calidad del servicio. La promoción de la salud en el trabajo no consiste solo en cumplir la normativa en materia de seguridad y salud en el trabajo. Es necesario también sensibilizar a las empresas e instituciones para que contribuyan a la mejora de sus empleados, y la forma de iniciar este proceso es realizar estudios dirigidos a la detección y evaluación de factores y riesgos psicosociales. En este sentido, no hay que olvidar la importancia fundamental de los trabajadores para las personas con discapacidad intelectual y es que, como señalan Devereux, Hastings y Noone (2009), el estudio de riesgos psicosociales, estrés y burnout en esta población es beneficioso 
PREVALENCIA Y NIVELES DE EXPOSICIÓN A FACTORES Y RIESGOS PSICOSOCIALES A TRAVÉS DEL ISTAS-21 ESMERALDA MONTALVO SÁNCHEZ, ELOÍSA GUERRERO BARONA, MARTA RODRÍGUEZ JIMÉNEZ, JONÁS AGUDO OSUNA, JUAN MANUEL MORENO MANSO Y DANIEL PAREDES GÓMEZ

para la organización; para el propio trabajador, que puede ver incrementado su bienestar, y, por último pero no menos importante, para la persona con discapacidad, ya que algunas investigaciones apuntan a que las interacciones entre el usuario con discapacidad y el trabajador que le proporciona apoyo podrían verse afectadas negativamente por el estrés del empleado.

\section{Método}

\subsection{Participantes y procedimiento}

El instrumento de evaluación fue entregado a una población de 727 trabajadores que prestan servicios de atención a personas con discapacidad intelectual o del desarrollo en Extremadura, de los cuales el $67,75 \%$ son mujeres y el $32,24 \%$ son hombres. La muestra final estuvo formada por 518 participantes de las diferentes entidades que representa el 71,25 \% de la muestra. El 30,11 \% son hombres (169) y el $67,37 \%$ mujeres (349). La media de edad se situó en torno a los 38,6 años $(D T=7,9)$ y la de experiencia laboral fue de 9,8 $(D T=6,8)$.

La unidad de gestión con mayor porcentaje de trabajadores son los centros ocupacionales $(19 \%)$, centros de día $(18 \%)$, residencias $(17 \%)$, centros educativos $(16 \%)$ y atención temprana (13\%). En el puesto de trabajo se especifica la función que desempeña cada trabajador. Los cuidadores (164) y los técnicos (107) representan el $31 \%$ y el $20 \%$ de la muestra, respectivamente. El $60 \%$ de los participantes tiene un contrato fijo, cuya antigüedad es de más de 10 años y el 80 \% de los sujetos no está satisfecho con su trabajo.

El instrumento de evaluación fue distribuido durante los meses de marzo a julio de 2013, entre 15 entidades de la Comunidad Autónoma de Extremadura dedicadas a la atención de personas con discapacidad intelectual o del desarrollo. La investigación ha sido llevada a cabo a través del convenio de colaboración entre la Universidad de Extremadura y FEAPS Extremadura. Los cuestionarios fueron repartidos en cada entidad participante por los gerentes o los delegados del comité de seguridad e higiene, se recogieron en sobres cerrados y fueron depositados en buzones ubicados en cada entidad, garantizando así la confidencialidad y siendo la colaboración voluntaria y anónima.

Algunas encuestas tuvieron que ser excluidas debido a que los cuestionarios se presentaron incompletos y este factor suponía una pérdida de información importante.

\subsection{Instrumento}

El método Istas-21 ha sido el instrumento utilizado para la evaluación de los factores y riesgos psicosociales en el presente trabajo, debido a su alta validez y fiabilidad (Gil-Monte, Carretero, Roldán, Desamparados y Núñez-Román, 2005). En España apareció, en 2003, CoPsoQ-istas21, como un instrumento de dominio 
PREVALENCIA Y NIVELES DE EXPOSICIÓN A FACTORES Y RIESGOS PSICOSOCIALES A TRAVÉS DEL ISTAS-21 ESMERALDA MONTALVO SÁNCHEZ, ELOÍSA GUERRERO BARONA, MARTA RODRÍGUEZ JIMÉNEZ, JONÁS AGUDO OSUNA, JUAN MANUEL MORENO MANSO Y DANIEL PAREDES GÓMEZ

público y uso gratuito. Posteriormente se publicó la 2. a versión (1.5), que mejoró la versión anterior y facilita el proceso de corrección e interpretación haciéndolo más claro (ISTAS, 2010).

Istas-21 consta de 120 preguntas estructuradas en cuatro partes: el contexto social y trabajo doméstico familiar (6 preguntas), salud y bienestar personal (26 preguntas), condiciones de empleo y trabajo (19 preguntas) y las dimensiones de las exposiciones psicosociales (69 preguntas). Las dimensiones recogidas en este instrumento son 20 y se encuentran a su vez agrupadas en los cinco constructos psicosociales (Tabla 1).

\begin{tabular}{|c|c|c|c|c|}
\hline $\begin{array}{l}\text { EXIGENCIAS } \\
\text { PSICOLÓGICAS }\end{array}$ & $\begin{array}{l}\text { CONTROL SOBRE } \\
\text { EL TRABAJO }\end{array}$ & $\begin{array}{l}\text { APOYO SOCIAL } \\
\text { Y CALIDAD DE } \\
\text { LIDERAZGO } \\
\end{array}$ & $\begin{array}{c}\text { COMPENSACIONES } \\
\text { DEL TRABAJO }\end{array}$ & $\begin{array}{c}\text { DOBLE } \\
\text { PRESENCIA }\end{array}$ \\
\hline $\begin{aligned} \text { - } & \text { Exigencias } \\
& \text { cuantitativas } \\
- & \text { Exigencia } \\
& \text { de esconder } \\
& \text { emociones } \\
- & \text { Exigencias } \\
& \text { emocionales } \\
- & \text { Exigencias } \\
& \text { cognitivas }\end{aligned}$ & $\begin{aligned} \text { - } & \text { Influencia } \\
\text { - } & \text { Posibilidades } \\
& \text { de desarrollo } \\
\text { - } & \text { Control sobre } \\
& \text { los tiempos a } \\
& \text { disposición } \\
\text { - } & \text { Sentido del } \\
& \text { trabajo } \\
\text { - } & \text { Compromiso }\end{aligned}$ & 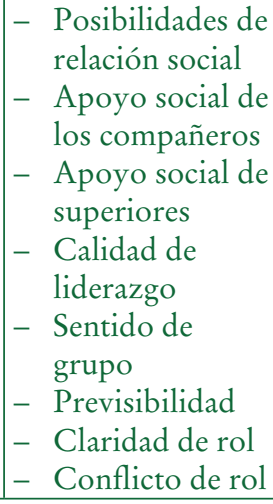 & $\begin{aligned} \text { - } & \text { Estima } \\
\text { - } & \text { Inseguridad } \\
& \text { sobre el futuro }\end{aligned}$ & $\begin{array}{l}- \text { Doble } \\
\text { presencia }\end{array}$ \\
\hline
\end{tabular}

Dada la extensión de los cuestionarios y las múltiples variables que comprende cada uno de los cinco constructos, decidimos centrarnos solo en tres de ellos, los que esperábamos que afectaban más a la población objeto de estudio: exigencias psicológicas, control sobre el trabajo y doble presencia. El primero, las exigencias psicológicas, tienen una doble vertiente: según la cantidad de trabajo (cuantitativa) y el tiempo disponible; o según la tarea que implique, es decir, trabajar con máquinas, herramientas o personas. Así, las exigencias psicológicas engloban (ISTAS, 2010): las exigencias emocionales, esconder emociones, cuantitativas y cognitivas. En primer lugar, las exigencias emocionales se refieren a la probabilidad de verse involucrado en la situación emocional con las demás personas del trabajo, tanto compañeros como clientes o usuarios. Por otro lado, las exigencias de esconder emociones hacen referencia a la capacidad de mantener una apariencia neutral independientemente del comportamiento de los usuarios o clientes, o con la relación de los compañeros o supervisores. Sin embargo, las exigencias cuantitativas se refieren a la relación entre la cantidad de trabajo y el tiempo disponible para realizarlo: volumen, ritmo, interrupciones e intensidad de trabajo. Y, finalmente, las exigencias cognitivas se refieren al manejo de conocimientos en relación a las posibilidades de desarrollo. Tienen que ver 
PREVALENCIA Y NIVELES DE EXPOSICIÓN A FACTORES Y RIESGOS PSICOSOCIALES A TRAVÉS DEL ISTAS-21 ESMERALDA MONTALVO SÁNCHEZ, ELOÍSA GUERRERO BARONA, MARTA RODRÍGUEZ JIMÉNEZ, JONÁS AGUDO OSUNA, JUAN MANUEL MORENO MANSO Y DANIEL PAREDES GÓMEZ

con el diseño y el contenido de tareas, su nivel de complejidad y el tiempo establecido para realizarlas.

El segundo constructo es el control sobre el trabajo, uno de los principales indicadores de buena salud. Podemos destacar que engloba las siguientes dimensiones (ISTAS, 2010): influencia, control del trabajo, sentido del trabajo, compromiso y posibilidad de desarrollo. La influencia es el margen de autonomía que se tiene en las tareas del trabajo y en los aspectos relacionados con la misma. El control del trabajo se aborda como la autonomía sobre los tiempos a disposición del individuo. En este sentido, se tiene en cuenta la organización temporal de la carga de trabajo, así como los descansos disponibles. Igualmente, hace referencia al sentido del trabajo como la relación de nuestro trabajo con otros valores como, por ejemplo, la utilidad del mismo o el aprendizaje, lo que favorecerá la realización de las tareas. En el caso del compromiso se refiere a la participación de los sujetos en las tareas desarrolladas y la implicación en las mismas. Y, finalmente, las posibilidades de desarrollo hacen referencia a las situaciones en las cuales el personal laboral puede poner en práctica los conocimientos y habilidades disponibles en función de su trabajo, al igual que la posibilidad de adquirir nuevos conocimientos.

El tercer constructo estudiado ha sido la doble presencia, entendida como la relación que existe entre las demandas del trabajo asalariado y del trabajo doméstico-familiar. Es decir, son las exigencias que establecen el trabajo y el ámbito doméstico-familiar. Son altas cuando las exigencias laborales influyen en las familiares (ISTAS, 2010).

\section{Resultados}

Los datos fueron analizados con un programa público y gratuito que se encuentra en la web de Istas, que son comparados con una muestra representativa de la población española, con un estudio llevado a cabo por Moncada, Llorens, Font, Galtés y Navarro (2005). En este estudio se establece la muestra de referencia para cada una de las dimensiones del cuestionario Istas-21.

Al tratarse de un estudio de prevalencia, se analizan la frecuencia y los porcentajes de los factores y riesgos psicosociales, concretamente de tres constructos: exigencias psicológicas, doble presencia y control del trabajo, así como sus correspondientes dimensiones.

\subsection{Exposición a factores psicosociales}

\subsubsection{Exigencias psicológicas}

En el constructo exigencias psicológicas la dimensión exigencias psicológicas emocionales es la que presenta un riesgo elevado para el 62,1\% de los individuos (Tabla 2). 
PREVALENCIA Y NIVELES DE EXPOSICIÓN A FACTORES Y RIESGOS PSICOSOCIALES A TRAVÉS DEL ISTAS-21 ESMERALDA MONTALVO SÁNCHEZ, ELOÍSA GUERRERO BARONA, MARTA RODRÍGUEZ JIMÉNEZ, JONÁS AGUDO OSUNA, JUAN MANUEL MORENO MANSO Y DANIEL PAREDES GÓMEZ

\begin{tabular}{|c|c|c|c|}
\hline \multicolumn{5}{|c|}{ TABLA 2. Porcentaje de profesionales en riesgo en las exigencias } \\
psicológicas por entidades
\end{tabular}

Los sujetos expresan tener un riesgo elevado en las exigencias psicológicas emocionales. Como se puede apreciar no existen diferencias entre hombres y mujeres en dichas exigencias. Lo mismo ocurre en función de la edad, exceptuando aquellos de más de 55 años, cuyo riesgo es menor (Tabla 3).

Las exigencias psicológicas emocionales afectan fundamentalmente a los cuidadores y responsables de área de las diferentes entidades, así como el personal de los centros ocupacionales. En función del contrato y de la antigüedad, el personal con mayor riesgo es el que tiene un contrato fijo con una antigüedad de más de 10 años (Tabla 3).

\begin{tabular}{|c|c|c|}
\hline \multicolumn{3}{|c|}{$\begin{array}{l}\text { TABLA 3. Porcentaje de trabajadores en riesgo en las variables estudiadas } \\
\text { en las exigencias emocionales }\end{array}$} \\
\hline & VARIABLES & EXIGENCIAS EMOCIONALES \\
\hline \multirow{2}{*}{ Sexo } & Hombres & $60,17 \%$ \\
\hline & Mujeres & $61,14 \%$ \\
\hline \multirow{5}{*}{ Edad } & Menos de 26 años & $45,6 \%$ \\
\hline & 26-35 años & $64,3 \%$ \\
\hline & 35-46 años & $57,7 \%$ \\
\hline & 46-55 años & $65,3 \%$ \\
\hline & Más de 55 años & $32,7 \%$ \\
\hline
\end{tabular}

Ediciones Universidad de Salamanca / CC BY-NC-ND

Siglo Cero, vol. 51 (1), 2020, enero-marzo, pp. 53-72

$$
-62-
$$


PREVALENCIA Y NIVELES DE EXPOSICIÓN A FACTORES Y RIESGOS PSICOSOCIALES A TRAVÉS DEL ISTAS-21

ESMERALDA MONTALVO SÁNCHEZ, ELOÍSA GUERRERO BARONA, MARTA RODRÍGUEZ JIMÉNEZ, JONÁS AGUDO OSUNA, JUAN MANUEL MORENO MANSO Y DANIEL PAREDES GÓMEZ

\section{TABLA 3. Porcentaje de trabajadores en riesgo en las variables estudiadas} en las exigencias emocionales (cont.)

\begin{tabular}{|c|c|c|}
\hline & VARIABLES & EXIGENCIAS EMOCIONALES \\
\hline \multirow{10}{*}{ Puesto de trabajo } & Responsable área & $79,56 \%$ \\
\hline & Personal administrativo & $24,4 \%$ \\
\hline & Técnico & $49,18 \%$ \\
\hline & Educación & $38,18 \%$ \\
\hline & Monitor & $51,9 \%$ \\
\hline & Fisioterapeuta & $31,6 \%$ \\
\hline & Cuidador & $55,9 \%$ \\
\hline & Personal cocina y limpieza & $32,14 \%$ \\
\hline & Mantenimiento & $3,3 \%$ \\
\hline & Conductor & $23,3 \%$ \\
\hline \multirow{10}{*}{ Departamento } & Centro educativo & $30,7 \%$ \\
\hline & Centro de día & $37,7 \%$ \\
\hline & Atención temprana & $36,7 \%$ \\
\hline & Centro ocupacional & $63,5 \%$ \\
\hline & Residencia & $23,35 \%$ \\
\hline & Admistración & $43,6 \%$ \\
\hline & Vivienda tutelada & $12,5 \%$ \\
\hline & Centro de Educación Especial & $4,1 \%$ \\
\hline & Ocio y Tiempo Libre & $8,3 \%$ \\
\hline & General-auxiliar & $18,3 \%$ \\
\hline \multirow{3}{*}{ Tipo de contrato } & Fijo & $64,1 \%$ \\
\hline & Discontinuo & $33,35 \%$ \\
\hline & Temporal & $47,2 \%$ \\
\hline \multirow{5}{*}{ Antigüedad } & Menos de 6 meses & $50 \%$ \\
\hline & 6-2 años & $12,8 \%$ \\
\hline & 2-5 años & $56,4 \%$ \\
\hline & 5-10 años & $59,4 \%$ \\
\hline & Más de 10 años & $72,37 \%$ \\
\hline
\end{tabular}

\subsubsection{Doble presencia}

El siguiente constructo analizado ha sido la doble presencia. Podemos destacar que el riesgo es elevado en todas las entidades participantes (Tabla 4). 
PREVALENCIA Y NIVELES DE EXPOSICIÓN A FACTORES Y RIESGOS PSICOSOCIALES A TRAVÉS DEL ISTAS-21 ESMERALDA MONTALVO SÁNCHEZ, ELOÍSA GUERRERO BARONA, MARTA RODRÍGUEZ JIMÉNEZ, JONÁS AGUDO OSUNA, JUAN MANUEL MORENO MANSO Y DANIEL PAREDES GÓMEZ

\begin{tabular}{|c|c|}
\hline $\begin{array}{c}\text { TABLA 4. Porcentaje de profesionales en riesgo } \\
\text { en la doble presencia por entidades }\end{array}$ \\
\hline ENTIDADES & DOBLE PRESENCIA \\
\hline A & $68,4 \%$ \\
\hline B & $75,8 \%$ \\
\hline C & $59,5 \%$ \\
\hline D & $66,7 \%$ \\
\hline E & $56,3 \%$ \\
\hline F & $88,9 \%$ \\
\hline G & $51,2 \%$ \\
\hline H & $70,8 \%$ \\
\hline I & $50 \%$ \\
\hline J & $63,9 \%$ \\
\hline L & $72,7 \%$ \\
\hline M & $64,3 \%$ \\
\hline N & $66,7 \%$ \\
\hline$\tilde{N}$ & $84,6 \%$ \\
\hline Total & $86,4 \%$ \\
\hline & $68,41 \%$ \\
\hline
\end{tabular}

Los resultados indican que las mujeres presentan un riesgo elevado en la doble presencia $(74,69 \%)$ frente a los varones (47,18 \%) (Tabla 5). Las personas más afectadas tienen entre 26 a 45 años, cuyos puestos de trabajo son responsables de área, administración, fisioterapeutas, cuidadores, personal de limpieza y cocina. Igualmente, esta dimensión provoca mayores riesgos en el personal de atención temprana, centro ocupacional, vivienda tutelada, administración, ocio y tiempo libre y general-auxiliar. En cuanto al tipo de contrato no se observan diferencias entre los/las profesionales, sin embargo, en relación a la antigüedad, los resultados indican que la mayoría de los sujetos están sometidos a un riesgo elevado (Tabla 5).

\begin{tabular}{|c|c|c|}
\hline \multicolumn{3}{|c|}{$\begin{array}{l}\text { TABLA 5. Porcentaje de profesionales en riesgo en las variables estudiadas } \\
\text { en la doble presencia }\end{array}$} \\
\hline & VARIABLES & DOBLE PRESENCIA \\
\hline \multirow{2}{*}{ Sexo } & Hombres & $47,18 \%$ \\
\hline & Mujeres & $74,69 \%$ \\
\hline \multirow{5}{*}{ Edad } & Menos de 26 años & $27,8 \%$ \\
\hline & 26-35 años & $66,20 \%$ \\
\hline & 35-46 años & $75,5 \%$ \\
\hline & 46-55 años & $48,3 \%$ \\
\hline & Más de 55 años & $45,1 \%$ \\
\hline
\end{tabular}

Ediciones Universidad de Salamanca / CC BY-NC-ND

Siglo Cero, vol. 51 (1), 2020, enero-marzo, pp. 53-72

$$
-64 \text { - }
$$


PREVALENCIA Y NIVELES DE EXPOSICIÓN A FACTORES Y RIESGOS PSICOSOCIALES A TRAVÉS DEL ISTAS-21

ESMERALDA MONTALVO SÁNCHEZ, ELOÍSA GUERRERO BARONA, MARTA RODRÍGUEZ JIMÉNEZ, JONÁS AGUDO OSUNA, JUAN MANUEL MORENO MANSO Y DANIEL PAREDES GÓMEZ

\section{TABLA 5. Porcentaje de profesionales en riesgo en las variables estudiadas} en la doble presencia (cont.)

\begin{tabular}{|c|c|c|}
\hline & VARIABLES & DOBLE PRESENCIA \\
\hline \multirow{10}{*}{ Puesto de trabajo } & Responsable área & $82,8 \%$ \\
\hline & Personal administrativo & $45,5 \%$ \\
\hline & Técnico & $69,27 \%$ \\
\hline & Educación & $39,68 \%$ \\
\hline & Monitor & $51,18 \%$ \\
\hline & Fisioterapeuta & $41,10 \%$ \\
\hline & Cuidador & $56,9 \%$ \\
\hline & Personal cocina y limpieza & $46,6 \%$ \\
\hline & Mantenimiento & $6,6 \%$ \\
\hline & Conductor & $10 \%$ \\
\hline \multirow{10}{*}{ Departamento } & Centro educativo & $29,5 \%$ \\
\hline & Centro de día & $44,61 \%$ \\
\hline & Atención temprana & $52,18 \%$ \\
\hline & Centro ocupacional & $69,31 \%$ \\
\hline & Residencia & $19,71 \%$ \\
\hline & Admistración & $48,54 \%$ \\
\hline & Vivienda tutelada & $18,05 \%$ \\
\hline & Centro de Educación Especial & $1,39 \%$ \\
\hline & Ocio y Tiempo Libre & $16,6 \%$ \\
\hline & General-auxiliar & $22,9 \%$ \\
\hline \multirow{3}{*}{ Tipo de contrato } & Fijo & $67,8 \%$ \\
\hline & Discontinuo & $33,35 \%$ \\
\hline & Temporal & $43,35 \%$ \\
\hline \multirow{5}{*}{ Antigüedad } & Menos de 6 meses & $17,14 \%$ \\
\hline & 6-2 años & $14,28 \%$ \\
\hline & 2-5 años & $61,25 \%$ \\
\hline & 5-10 años & $66,38 \%$ \\
\hline & Más de 10 años & $77,32 \%$ \\
\hline
\end{tabular}

\subsubsection{Control del trabajo}

En el análisis del constructo control del trabajo puede observarse que existe mayor riesgo en la dimensión de control de los tiempos, de manera que un 70,36 \% del personal presenta riesgo elevado (Tabla 6). 
PREVALENCIA Y NIVELES DE EXPOSICIÓN A FACTORES Y RIESGOS PSICOSOCIALES A TRAVÉS DEL ISTAS-21 ESMERALDA MONTALVO SÁNCHEZ, ELOÍSA GUERRERO BARONA, MARTA RODRÍGUEZ JIMÉNEZ, JONÁS AGUDO OSUNA, JUAN MANUEL MORENO MANSO Y DANIEL PAREDES GÓMEZ

\begin{tabular}{|c|c|c|c|c|}
\hline \multicolumn{5}{|c|}{$\begin{array}{c}\text { TABLA 6. Porcentaje de profesionales en riesgo en el control del trabajo } \\
\text { por entidades }\end{array}$} \\
\hline INFLUENCIA & $\begin{array}{l}\text { POSIBILIDAD } \\
\text { DE DESARROLLO }\end{array}$ & CONTROL TIEMPO & SENTIDO TRABAJO & COMPROMISO \\
\hline $35 \%$ & $5 \%$ & $80 \%$ & $5 \%$ & $20 \%$ \\
\hline $27,3 \%$ & $22,6 \%$ & $58,1 \%$ & $6,5 \%$ & $19,4 \%$ \\
\hline $40,5 \%$ & $4,8 \%$ & $85,7 \%$ & $2,4 \%$ & $17,1 \%$ \\
\hline $18,4 \%$ & $6 \%$ & $63,3 \%$ & $0 \%$ & $6 \%$ \\
\hline $37,5 \%$ & $12,5 \%$ & $75 \%$ & $6,3 \%$ & $0 \%$ \\
\hline $55,6 \%$ & $0 \%$ & $87,5 \%$ & $0 \%$ & $22,2 \%$ \\
\hline $47,7 \%$ & $19,6 \%$ & $80,4 \%$ & $4,3 \%$ & $4,3 \%$ \\
\hline $40,3 \%$ & $18,2 \%$ & $96,9 \%$ & $7,6 \%$ & $26,2 \%$ \\
\hline $65,7 \%$ & $20 \%$ & $80 \%$ & $2,9 \%$ & $12,1 \%$ \\
\hline $37,7 \%$ & $9,5 \%$ & $79,4 \%$ & $4,7 \%$ & $2,8 \%$ \\
\hline $20 \%$ & $0 \%$ & $20 \%$ & $0 \%$ & $0 \%$ \\
\hline $20,7 \%$ & $3,4 \%$ & $64,3 \%$ & $3,4 \%$ & $6,9 \%$ \\
\hline $22,2 \%$ & $11,1 \%$ & $77,8 \%$ & $22,2 \%$ & $44,4 \%$ \\
\hline $14,3 \%$ & $7,7 \%$ & $35,7 \%$ & $7,1 \%$ & $21,4 \%$ \\
\hline $54,5 \%$ & $18,2 \%$ & $81 \%$ & $13,6 \%$ & $18,2 \%$ \\
\hline $35,8 \%$ & $10,57 \%$ & $70,36 \%$ & $5,7 \%$ & $14,7 \%$ \\
\hline
\end{tabular}

En conjunto, el control del tiempo es una dimensión de riesgo elevado en la mayoría de los sujetos, afectando a los dos sexos, aunque las mujeres tienen un nivel algo más elevado $(72,55 \%$ ) frente a los varones $(63,22 \%$ ) (Tabla 7$)$. El grupo de edad de 26 a 35 años expresa tener dificultades para el control del tiempo en su puesto de trabajo y todo el personal perteneciente a los diferentes departamentos está afectado por el control del tiempo. También afecta a todos los tipos de contrato y no está condicionado por la antigüedad laboral (Tabla 7).

\section{TABLA 7. Porcentaje de profesionales en riesgo en las variables estudiadas en el control del tiempo}

\begin{tabular}{|c|c|c|}
\hline & VARIABLES & CONTROL DEL TIEMPO \\
\hline \multirow{3}{*}{ Sexo } & Hombres & $63,22 \%$ \\
\cline { 2 - 3 } & Mujeres & $72,55 \%$ \\
\hline \multirow{4}{*}{ Edad } & Menos de 26 años & $45 \%$ \\
\cline { 2 - 3 } & $26-35$ años & $77,03 \%$ \\
\cline { 2 - 3 } & $35-46$ años & $63,64 \%$ \\
\cline { 2 - 3 } & $46-55$ años & $52,6 \%$ \\
\cline { 2 - 3 } & Más de 55 años & $40,93 \%$ \\
\hline
\end{tabular}


PREVALENCIA Y NIVELES DE EXPOSICIÓN A FACTORES Y RIESGOS PSICOSOCIALES A TRAVÉS DEL ISTAS-21

ESMERALDA MONTALVO SÁNCHEZ, ELOÍSA GUERRERO BARONA, MARTA RODRÍGUEZ JIMÉNEZ, JONÁS AGUDO OSUNA, JUAN MANUEL MORENO MANSO Y DANIEL PAREDES GÓMEZ

\section{TABLA 7. Porcentaje de profesionales en riesgo en las variables estudiadas en el control del tiempo (cont.)}

\begin{tabular}{|c|c|c|}
\hline & VARIABLES & CONTROL DEL TIEMPO \\
\hline \multirow{10}{*}{ Puesto de trabajo } & Responsable área & $41,44 \%$ \\
\hline & Personal administrativo & $11,76 \%$ \\
\hline & Técnico & $69,94 \%$ \\
\hline & Educación & $52,23 \%$ \\
\hline & Monitor & $56,89 \%$ \\
\hline & Fisioterapeuta & $43,33 \%$ \\
\hline & Cuidador & $69,59 \%$ \\
\hline & Personal cocina y limpieza & $55 \%$ \\
\hline & Mantenimiento & $26,66 \%$ \\
\hline & Conductor & $23,33 \%$ \\
\hline \multirow{10}{*}{ Departamento } & Centro educativo & $43,82 \%$ \\
\hline & Centro de día & $50,34 \%$ \\
\hline & Atención temprana & $58,56 \%$ \\
\hline & Centro ocupacional & $66,05 \%$ \\
\hline & Residencia & $27,67 \%$ \\
\hline & Administración & $21,87 \%$ \\
\hline & Vivienda tutelada & $23,95 \%$ \\
\hline & Centro de Educación Especial & $5,55 \%$ \\
\hline & Ocio y Tiempo Libre & $25 \%$ \\
\hline & General-auxiliar & $48,33 \%$ \\
\hline \multirow{3}{*}{ Tipo de contrato } & Fijo & $73,65 \%$ \\
\hline & Discontinuo & $50 \%$ \\
\hline & Temporal & $94,45 \%$ \\
\hline \multirow{5}{*}{ Antigüedad } & Menos de 6 meses & $40 \%$ \\
\hline & 6-2 años & $47,14 \%$ \\
\hline & 2-5 años & $81,15 \%$ \\
\hline & 5-10 años & $80,42 \%$ \\
\hline & Más de 10 años & $67,52 \%$ \\
\hline
\end{tabular}

\section{Discusión}

El objetivo principal que se ha planteado en este estudio exploratorio, descriptivo y de prevalencia ha sido analizar los factores y riesgos psicosociales de los sujetos que atienden a personas con discapacidad intelectual o del desarrollo. Los resultados 
PREVALENCIA Y NIVELES DE EXPOSICIÓN A FACTORES Y RIESGOS PSICOSOCIALES A TRAVÉS DEL ISTAS-21

ESMERALDA MONTALVO SÁNCHEZ, ELOÍSA GUERRERO BARONA, MARTA RODRÍGUEZ JIMÉNEZ, JONÁS AGUDO OSUNA, JUAN MANUEL MORENO MANSO Y DANIEL PAREDES GÓMEZ

obtenidos indican que existen indicios de riesgos y factores psicosociales en este grupo de sujetos, por ello es importante llevar a cabo estudios más rigurosos para la prevención y eliminación de estos riesgos (Moreno-Jiménez y Garrosa, 2013).

Sin embargo, el análisis de los tres constructos psicosociales que engloban 10 dimensiones del cuestionario indica que el personal está expuesto a factores y riesgos psicosociales que perjudican la salud del personal. Las dimensiones con mayor porcentaje de individuos en riesgo son control del tiempo, doble presencia, exigencias psicológicas emocionales y exigencias psicológicas cognitivas.

La dimensión con mayor riesgo para los participantes de este estudio es el control del trabajo. Constatamos que el 70,36 \% de los/las participantes expresan tener dificultades en la autonomía para controlar el tiempo en su puesto de trabajo, siendo este aspecto muy desfavorable para la salud, como han puesto de manifiesto los trabajos de Castillo, Santana, Valeta, Alvis y Romero (2011). Las mujeres de 26 a 35 años que son cuidadoras y técnicas de los centros ocupacionales expresan tener grandes dificultades para el control del tiempo en su puesto de trabajo. Igualmente, aquellos/ as profesionales con un contrato temporal están en riesgo elevado; sin embargo, en la variable de la antigüedad el perfil con más dificultades es el personal con más de 10 años de trabajo.

De la misma forma, los resultados muestran que la doble presencia supone un riesgo elevado para el 68,41 \% de las mujeres, coincidiendo con los trabajos de Caraballo y Blanco (2012). Afecta principalmente a las mujeres de 26 a 46 años. El puesto de trabajo más sensible a la doble presencia es el responsable de área y técnicos que trabajan en centros ocupacionales con un contrato fijo y la antigüedad de más de10 años.

Igualmente, los resultados muestran que todas las exigencias psicológicas (emocionales, cognitivas, cuantitativas y esconder emociones) presentan un porcentaje elevado de riesgo. Las exigencias de esconder emociones suponen un riesgo para el 62,1\% de los/las participantes, es decir, presenta dificultades para controlar las emociones en sus puestos de trabajo (Caballero-Arias y Blanco, 2012). Las exigencias cognitivas afectan de forma directa e indiferenciada a hombres y mujeres de 35 a 46 años que son responsables de área y técnicos, con un contrato fijo y una antigüedad de 2 a 10 años. Del mismo modo, las exigencias emocionales suponen un riesgo elevado para profesionales de ambos sexos, cuyas edades son de 26 a 35 años y de 46 a 55. Influye directamente en los cuidadores/cuidadoras y responsables de área que trabajan en centros ocupacionales. Su situación laboral es fija con una antigüedad de más de 10 años.

Por consiguiente, los sujetos que presentan altas exigencias psicológicas están expuestos a un mayor riesgo para su salud, como por ejemplo síntomas depresivos, que aquellos que expresan bajas demandas psicológicas (Ansoleaga y Toro, 2010).

De forma general, podemos concluir que no hay diferencias elevadas entre los hombres y las mujeres en el análisis de los tres constructos. Los responsables de área y cuidadores son los/las profesionales que mayor riesgo presentan, así como el personal fijo que lleva más de 5 años en las entidades. Los sujetos con mayor responsabilidad en sus puestos de trabajo presentan puntaciones más elevadas en los constructos estudiados (Castillo et al., 2011). Algunos estudios han detectado que las principales dimensiones problemáticas para estos/as profesionales son doble presencia, las 
PREVALENCIA Y NIVELES DE EXPOSICIÓN A FACTORES Y RIESGOS PSICOSOCIALES A TRAVÉS DEL ISTAS-21

ESMERALDA MONTALVO SÁNCHEZ, ELOÍSA GUERRERO BARONA, MARTA RODRÍGUEZ JIMÉNEZ, JONÁS AGUDO OSUNA, JUAN MANUEL MORENO MANSO Y DANIEL PAREDES GÓMEZ

exigencias psicológicas, el control del tiempo, inseguridad, previsibilidad, calidad de liderazgo y estima (Otero y Dalmau, 2007). Estos resultados son coherentes con los obtenidos en nuestro estudio.

Los resultados indican que los individuos que no tienen autonomía en el control del tiempo van a tener más posibilidades de sufrir estrés, incluso tienen una percepción negativa de su salud (Cladellas y Casteló, 2012). La dimensión del control del tiempo tiene una mayor relevancia en las mujeres con cargas familiares que en los hombres, aunque no existen diferencias significativas entre ambos (Cladellas y Casteló, 2012). Esta falta de autonomía en el control del tiempo y del trabajo influye en la satisfacción laboral del personal laboral (Grau-Alberola, 2014). Curiosamente en varones la percepción de la salud positiva aumenta cuando tienen un control de su trabajo y del sentido del mismo, mientras que en las mujeres la salud se relaciona con la posibilidad de desarrollo de la carrera y de relación social ('Tomás, Sancho, Navarro y Meléndez, 2009).

Para finalizar, quisiéramos exponer algunas de las limitaciones de la investigación, ya que se trata de un estudio exploratorio, descriptivo y de prevalencia realizado con el Istas-21. Una de las principales limitaciones es que los datos se obtuvieron a través de medidas de autoinforme y metodología transversal, por lo que las relaciones entre variables pueden estar sesgadas, dificultando extraer relaciones causales. Además, se requieren instrumentos más precisos para valorar riesgos psicosociales más específicos, como por ejemplo trabajo emocional, estrés laboral, apoyo social, entre otros. Tampoco se realizó ningún tipo de muestreo para la selección de los sujetos, aunque sí hemos obtenido unos índices muy elevados de participación de las entidades extremeñas. En este estudio solo se han analizado 10 de las 20 dimensiones del cuestionario, esperamos en un futuro extraer conclusiones del resto, aunque algunos de los datos generales expuestos, por ejemplo, que un $80 \%$ de los participantes no está satisfecho con el trabajo que realizan, puede considerarse indicador de riesgos e iniciarse medidas preventivas urgentes. Asimismo, este trabajo permite identificar las entidades y/o asociaciones más vulnerables de riesgos psicosociales y adoptar estrategias que minimicen sus efectos.

Para futuros estudios, nos proponemos realizar una investigación más exhaustiva y de corte longitudinal en los trabajadores que atienden a personas con discapacidad intelectual o del desarrollo para examinar los cambios en las relaciones entre variables que se analizan. Sin embargo, pese a las limitaciones, este trabajo, además, nos ha estimulado a profundizar en algunos riesgos específicos en un estudio sobre calidad de vida laboral ya iniciado y esperamos, además, contribuir con este estudio pionero en nuestra comunidad a detectar factores de riesgos psicosociales en el personal que presta servicios de atención directa a personas con discapacidad intelectual o del desarrollo.

\section{Referencias bibliográficas}

Agencia Europea para la Seguridad y la Salud en el Trabajo (2009). Encuesta Europea en las empresas sobre riesgos nuevos y emergentes, ESENER. Observatorio Europeo de Riesgos. Recuperado de https://osha.europa.eu/es/esener-enterprise-survey/enterprisesurvey-esener. 
PREVALENCIA Y NIVELES DE EXPOSICIÓN A FACTORES Y RIESGOS PSICOSOCIALES A TRAVÉS DEL ISTAS-21 ESMERALDA MONTALVO SÁNCHEZ, ELOÍSA GUERRERO BARONA, MARTA RODRÍGUEZ JIMÉNEZ, JONÁS AGUDO OSUNA, JUAN MANUEL MORENO MANSO Y DANIEL PAREDES GÓMEZ

Agencia Europea para la Seguridad y la Salud en el Trabajo (2013). Sondeo de opinión paneuropeo sobre seguridad y salud en el trabajo. Recuperado de https://osha.europa.eu/ es/safety-health-in-figures.

Ansoleaga, E. y Toro, J. P. (2010). Factores psicosociales laborales asociados a riesgo de sintomatología depresiva en trabajadores de una empresa minera. Salud de los Trabajadores, 18, 1. Recuperado de http://www.scielo.org.ve/scielo.php?pid=S1315-01382010000100002\&script=sci_ arttext.

Avargues, M. L., Borda, M. y López, A. M. (2010). Condiciones de trabajo, Burnout y síntomas de estrés en la universidad: validación de un modelo estructural sobre el efecto mediador de la competencia personal percibida. Psicología Conductual, 18(1), 317-341.

Caraballo-Arias, Y. y Blanco, G. (2012). Identificación y evaluación de factores psicosociales laborales en un centro de atención de llamadas. Revista de la Facultad de Medicina, 35(2), 21-27.

Castillo, I. Y., Santana, M., Valeta, A., Alvis, L. y Romero, E. (2011). Factores de riesgo psicosociales del trabajo en médicos de una Empresa Social del Estado en Cartagena de Indias, Colombia. Revista de la Facultad Nacional de Salud Pública, 29(4), 363-371.

Cladellas, R. y Castelló, A. (2011). Percepción del estado de Salud y estrés, de profesores universitarios, en relación con la franja horaria de docencia. Electronic Journal of Research in Educational Psychology, 9(1), 217-240. Recuperado de http://www.redalyc.org/articulo. oa? id=293122834011.

Devereux, J., Hastings, R. y Noone, S. (2009). Staff stress and burnout in intellectual disability services: work stress theory and its application. Journal of Applied Research in Intellectual Disabilities, 22(6), 561-573.

Figueiredo-Ferraz, H., Gil-Monte, P. R. y Grau, E. (2009). Prevalencia del síndrome quemarse por el trabajo (Burnout) en una muestra de maestros portugueses. Aletheia, 29, 6-15.

Forastieri, V. (2012). ¿Por qué es importante el estrés relacionado con el trabajo? La acción de la OIT y el "enfoque solve". En Observatorio de Riesgos Psicosociales UGT, Anuario internacional sobre prevención de riesgos psicosociales y calidad de vida en el trabajo (pp. 117-136). Secretaría General de Salud Laboral UGT-CEC. Recuperado de http:// www.ugt.es/actualidad/2012/octubre/AnuarioSlaboral2012Web.pdf.

Gil-Monte, P. R. (2009). Algunas razones para considerar los riesgos psicosociales en el trabajo y sus consecuencias en la salud pública. Revista Española de Salud Pública, 83(2), 169173. Recuperado de http://www.redalyc.org/articulo.oa?id=36323272012.

Gil-Monte, P. R. (2012). Riesgos psicosociales en el trabajo y salud ocupacional. Revista Peruana de Medicina Experimental y Salud Publica, 29(2), 237-341. Recuperado de http:// www.redalyc.org/articulo.oa?id=36323272012.

Gil-Monte, P. (2014). Manual de psicosociología aplicada al trabajo y a la prevención de los riesgos laborales. Madrid: Pirámide.

Gil-Monte, P., Carretero, N., Roldán, M. D. y Núñez-Román, E. (2005). Prevalencia del síndrome de quemarse en el trabajo (burnout) en monitores de taller para personas con discapacidad. Revista de Psicología del Trabajo y de las Organizaciones, 21(1-2), 107-123.

Gil-Monte, P. R. y Figueiredo-Ferraz, H. (2013). Psychometric properties of the "Spanish Burnout Inventory" among employees woorking with people with intellectual disability. Journal of Intellectual Disability Research, 57(10), 959-968. Recuperado de http://www. uv.es/unipsico/instrumentos-de-evaluacion/.

Grau-Alberola, E. (2014). Conflicto y conciliación trabajo-familia. En P. R. Gil-Monte (Coord.), Manual de psicosociología aplicada al trabajo y a la prevención de los riesgos psicosociales. Madrid: Pirámide.

Ediciones Universidad de Salamanca / CC BY-NC-ND

Siglo Cero, vol. 51 (1), 2020, enero-marzo, pp. 53-72 
PREVALENCIA Y NIVELES DE EXPOSICIÓN A FACTORES Y RIESGOS PSICOSOCIALES A TRAVÉS DEL ISTAS-21 ESMERALDA MONTALVO SÁNCHEZ, ELOÍSA GUERRERO BARONA, MARTA RODRÍGUEZ JIMÉNEZ, JONÁS AGUDO OSUNA, JUAN MANUEL MORENO MANSO Y DANIEL PAREDES GÓMEZ

Gray-Stanley, J. y Muramatsu, N. (2011). Work stress, burnout, and social and personal resources among direct care workers. Research in Developmental Disabilities, 32(3), 1065-1074.

Guerrero, E., Gómez, R., Moreno, J. M., García-Baamonde, E. y Blázquez, M. (2011). El síndrome de quemado, modos de afrontamiento del estrés y salud mental en profesores no universitarios. Psicología Conductual, 19(3), 557-576.

INSHT (1995). Artículo 4.7. de la Ley 31/1995, de 8 de noviembre, de Prevención de Riesgos Laborales. $B O E, 269,32590-32611$. Madrid: Autor. Recuperado de https:/www.boe.es/ buscar/act.php?id=BOE-A-1995-24292.

INSHT (2012). VII Encuesta Nacional de condiciones de trabajo. Madrid: Instituto Nacional de Seguridad e Higiene en el Trabajo.

Instituto Sindical de Trabajo, Ambiente y Salud, ISTAS (2010). Manual del método CoPsoQ-istas21 (versión 1.5) para la evaluación y prevención de los riesgos psicosociales para empresas con 25 o más trabajadores y trabajadoras. Recuperado de http://www.istas.net/ copsoq/ficheros/documentos/manual_metodo.pdf.

Kozak, A., Kersten, M., Schillmöller, Z. y Nienhaus, A. (2013). Psychosocial workrelated predictors and consequences of personal burnout among staff working with people with intellectual disabilities. Research in Developmental Disabilities, 34(1), 102-115.

Limonero, J. T., Tomás-Sábado, J., Fernández-Castro, J., Gómez-Romero, M. J. y ArdiLla-Herrero, A. (2012). Estrategias de afrontamiento resilientes y regulación emocional: predictores de satisfacción con la vida. Psicología Conductual, 20(1), 183-196.

Mansilla, F., García, J. C., Gamero, C. y Congosto, A. (2010). Influencia de la insatisfacción laboral en las demandas de cambio de puesto de trabajo por motivos de salud. Medicina y Seguridad en el Trabajo, 56, 147-157.

Moncada, S., Llorens, C., Font, A., Galtés, A. y Navarro, A. (2008). Exposición a riesgos psicosociales entre la población asalariada en España (2004-05): valores de referencia de las 21 dimensiones del cuestionario COPSOQ Istas21. Revista Española de Salud Pública, 82(6), 667-675.

Moreno, B. y Báez, C. (2010). Factores y riesgos psicosociales, formas, consecuencias, medidas y buenas prácticas. Madrid: Instituto Nacional de Seguridad e Higiene en el Trabajo.

Moreno-Jiménez, B. y Garrosa, E. (2013). Salud Laboral. Riesgos laborales psicosociales y bienestar laboral. Madrid: Pirámide.

Moriana, J. A., Alós, F., Pino, M. J., Herruzo, J., Ruiz, R., Alcalá, R. y Corpas, A. J. (2006). Análisis y comparación del nivel de Burnout en dos profesiones asistenciales. Psicología Conductual, 14(1), 7-17.

Mutkins, E., Brown, R. F. y Thorsteinsson, E. (2011). Stress, depression, workplace and social supports and burnout in intellectual disability support staff. Journal of Intellectual Disability Research, 55(5), 500-510.

Oscal, A., López-Araujo, B., Bardera, P., Uríen, B., Díez, V. y Rubio, C. (2014). Riesgos psicosociales y accidentabilidad laboral: investigación y propuestas de actuación. Papeles del Psicólogo, 35(2), 138-143. Recuperado de http://www.papelesdelpsicologo.es/pdf/2365. pdf.

Otero, C. y Dalmau, I. (2007). Métodos de Evaluación de Factores Psicosociales en el Sector Sanitario, FSICO E ISTAS21. Instituto Nacional de Seguridad e Higiene en el Trabajo. Recuperado de http://aulavirtual2000.com/curso_basico/documents/HOSPITAL-PFSICOISTAS21.pdf.

Peiró, J., Yeves, J. y Llorente, L. (2013). El análisis de los riesgos psicosociales en el trabajo: investigación y práctica profesional en España. En Observatorio de Riesgos Psicosociales 
PREVALENCIA Y NIVELES DE EXPOSICIÓN A FACTORES Y RIESGOS PSICOSOCIALES A TRAVÉS DEL ISTAS-21 ESMERALDA MONTALVO SÁNCHEZ, ELOÍSA GUERRERO BARONA, MARTA RODRÍGUEZ JIMÉNEZ, JONÁS AGUDO OSUNA, JUAN MANUEL MORENO MANSO Y DANIEL PAREDES GÓMEZ

UGT, Anuario internacional sobre prevención de riesgos psicosociales y calidad de vida en el trabajo (pp. 20-46). Secretaría General de Salud Laboral y Medio Ambiente: UGT-CEC. Recuperado de http://www.ugt.es/saludlaboral/observatorio/publicaciones/new2013/ Anuario2013ESP.pdf.

Ramos, J. y Peiró, J. (2014). La psicología del trabajo y las organizaciones en tiempos de crisis económica. Papeles del Psicólogo, 35(1), 1-4.

Salanova, M. y Llorens, S. (2008). Estado actual y retos futuros en el estudio de burnout. Papeles del Psicólogo, 29, 59-67.

Salanova, M., Peiró, J. M. y Schaufeli, W. (2001). Selef-efficacy specificity and Burnout among informatiom technology workers: an extensión of the Job Demands-Control Model. European Journal on Work and Organizational Psychology, 11, 89-97.

Schaufeli, W. y Salanova, M. (2002). La evaluación de riesgos psicosociales en el trabajo. Revista Prevención, Trabajo y Salud, 20, 4-9.

Tomás, J. M., Sancho, P., Navarro, E. y Meléndez, J. C. (2009) Relaciones entre factores psicosociales y salud en función del sexo. Ansiedad y Estrés, 15(1), 1-11. 